\title{
Proust et l'autofiction : vers un montage des identités
}

\author{
Thomas Carrier-Lafleur \\ Université Laval et Université Paul-Valéry (Montpellier III)
}

Bientôt je pus montrer quelques esquisses. Personne n'y comprit rien. Même ceux qui furent favorables à ma perception des vérités que je voulais ensuite graver dans le temple, me félicitèrent de les avoir découvertes au "microscope», quand je m'étais au contraire servi d'un télescope pour apercevoir des

choses, très petites en effet, mais parce qu'elles étaient situées à une grande distance, et qui étaient chacune un monde. Là où je cherchais les grandes lois, on m'appelait fouilleur de détails. D'ailleurs, à quoi bon faisais-je cela? J'avais eu de la facilité, jeune, et Bergotte avait trouvé mes pages de collégien « parfaites ». Mais au lieu de travailler j'avais vécu dans la paresse, dans la dissipation des plaisirs, dans

la maladie, les soins, les manies, et j'entreprenais mon ouvrage à la veille de mourir, sans rien savoir de mon métier.

Marcel Proust, Le Temps retrouvé 
La matrice «autofiction» est née sous la plume de Serge Doubrovsky, en 1977, sur la quatrième de couverture de son roman Fils (ces lignes explicatives seront reprises pour la réédition de Fils chez Gallimard, cette fois dans la préface). Bien que le néologisme, suivant l'évolution de l'œuvre de Doubrovsky, ait connu plusieurs altérations sémantiques, on peut encore aujourd'hui se référer à la définition première du terme. L'auteur, expliquant l'énoncé de Philippe Gasparini ${ }^{1}$ qui avance que toute définition de l'autofiction passe d'abord par une critique de l'autobiographie (ce qui confirme malgré tout l'analogie entre ces deux types d'énonciation intime ou intimiste), écrit d'emblée: "Autobiographie? Non, c'est un privilège réservé aux importants de ce monde, au soir de leur vie, et dans un beau style. » (Doubrovsky, 2001, p. 10) Entre les lignes, on comprend que l'autofiction serait une sorte d'autobiographie des inconnus: nous sommes tous des inconnus, c'est-à-dire des êtres fictifs, en comparaison avec un Jean-Jacques Rousseau qui écrit ses Confessions. S'ensuit la définition à proprement parler : «Fiction, d'événements et de faits strictement réels ; si l'on veut, autofiction, d'avoir confié le langage d'une aventure à l'aventure du langage, hors sagesse et hors syntaxe du roman, traditionnel ou nouveau » (p. 10). Par la suite, le terme tomba dans l'oubli pour mieux réapparaître lors du débat entourant la parution du Livre brisé, l'ouvrage le plus important de Doubrovsky, au début des années 1990. Parallèlement, Gérard Genette et Vincent Colonna proposent une autre définition du néologisme doubrovskien, faisant

\footnotetext{
1 Au cours de la conférence «Autofiction vs Autobiographie » qu'il a présentée au colloque «Enjeux critiques des écritures (auto)biographiques contemporaines » organisé les 10 et 11 mai 2010 à l'Université de Montréal dans la cadre du 78e Congrès de l'Acfas.
} 
passer la fiction - du côté de la forme chez l'auteur de Fils du côté du contenu. Colonna, par exemple, invente le concept de «fictionnalisation de soi » pour, ensuite, le renommer, coupant l'herbe sous le pied à Doubrovsky, autofiction :

La fictionnalisation de soi consiste à s'inventer des aventures que l'on s'attribuera, à donner son nom d'écrivain à un personnage introduit dans des situations imaginaires [...] il faut que l'écrivain ne donne pas à cette intention une valeur figurale ou métaphorique, qu'il n'encourage pas une lecture référentielle qui déchiffrerait dans le texte des confidences indirectes. (1989, p. 10)

Depuis ce temps, la poétique moderne oscille entre ces deux axes, hésite entre les deux pôles de l'autofiction.

Dans cet article, nous interrogerons l'auteur qui nous semble le mieux incarner ce que serait la formule d'une autofiction avant la lettre, à savoir Marcel Proust avec $\grave{A}$ la recherche du temps perdu. On peut déjà noter que Doubrovsky, Genette et Colonna se sont questionnés sur Proust et sur son rapport à l'autofiction. D'une part Doubrovsky, dans certaines pages de critique littéraire (pensons à l'article portant sur le début du Côté de chez Swann, "Corps du texte/texte du corps »), mais plus précisément, sous la forme d'une filiation, dans l'ouvrage Un amour de soi, titre qui reprend le Un amour de Swann de l'auteur de la Recherche. D'autre part Genette, dans Palimpsestes, avec un échange de lettres entre Proust et Mme Scheikévitch où Proust fait une sorte de sommaire - à la première personne - du livre à venir, À l'ombre des jeunes filles en fleurs, confirmant la possible identification onomastique entre l'auteur Marcel Proust et le narrateur de la Recherche. Pour définir le « contrat de lecture » que Proust propose avec ce passage, Genette dit que «le meilleur terme serait sans doute 
celui avec lequel Doubrovsky désigne son propre récit: autofiction " (Genette, 1982, p. 358). Finalement, Colonna, vers la fin de sa thèse, affirme que «c'est évidemment à la Recherche [...] de Marcel Proust que nous devons la plupart des autofictions contemporaines » (1989, p. 324), pour conclure avec ceci : " Du centre Proust, essaiment ainsi la plupart des autofictions modernes » (p. 328).

Nous croyons que le rapport de Proust avec l'autofiction dépasse le duel entre la forme et le contenu, les pourparlers entre Doubrovsky et Genette-Colonna. Pour sa Recherche, Proust avait besoin de l'autofiction, ou, plutôt, il avait besoin d'une forme d'énonciation personnelle complètement moderne qui, quelques décennies plus tard, sera nommée autofiction. Pour le démontrer, nous traiterons d'abord de la (curieuse) nature de l'apprentissage littéraire du narrateur proustien et du rapport qu'il entretient avec les déceptions. Du coup, nous étudierons la crise existentielle qui frappe ce narrateur et comment la découverte d'une nouvelle forme littéraire, l'autofiction, l'empêche de sombrer dans le nihilisme moderne. En flirtant avec l'autofiction, Proust propose une nouvelle forme de montage littéraire, le montage des identités.

\section{Proust et les déceptions : à la recherche d'une autofiction}

Dans Proust et les horreurs de l'amour, Nicolas Grimaldi souligne: "À la recherche du temps perdu est le roman des déceptions », car " [t] out le but de l'entreprise est de répondre à cette unique question. Connaîtrons-nous jamais la réalité? Trouverons-nous jamais aucun moyen de nous unir à elle? » (2008, p. 9 et 11). Les déceptions structurent l'anatomie de la 
Recherche, d'un point de vue aussi bien narratif qu'esthétique ou théorique, le tout à travers l'apprentissage du hérosnarrateur. Cette thématique des déceptions proustiennes jongle avec des notions vitales : l'amour, la solitude, l'habitude, le réel, la vie, la mémoire, l'expérience, le monde, l'individu. C'est pourquoi il faut y percevoir des rapports indéniables avec l'autofiction, son esthétique, sa poétique, sa raison d'être. Avec l'autofiction, les deux côtés se rejoignent enfin : le côté de l'art et le côté de la vie. Et les misères d'une vie peuvent alors devenir les splendeurs d'une œuvre d'art. L'apprentissage du narrateur se doit d'aboutir, d'une manière anachronique, sur le néologisme doubrovskien. Dans cette optique, un passage précis de la Recherche doit être éclairé : celui de la lecture, par le narrateur, du Journal des frères Goncourt. À travers ce pastiche des deux grands écrivains et mémorialistes, Proust laisse entrevoir le fil rouge de son esthétique, la modernité de son œuvre.

\section{Le Journal inédit des Goncourt}

Ce passage n'a pas l'exaltation des autres séquences célèbres qui rythment l'apprentissage du héros proustien vers sa carrière d'homme de lettres : l'expérience de la madeleine, le côté romanesque de la soirée chez les Guermantes, la violence de la vie en commun avec Albertine ou encore les rêveries et le drame propres au séjour à Venise. Il s'agit toutefois d'un moment crucial dans son apprentissage, car il est en lien direct avec le problème de la littérature.

Contrairement aux quatre segments mentionnés, dans celui du Journal, la déception ne vient pas après quelques 
sentiments heureux, elle arrive avant: elle n'a donc pas le dernier mot. Le narrateur est plus lucide face à sa déception, va même jusqu'à l'analyser, ce qui modèlera la suite de son apprentissage, le sillage de sa vocation d'écrivain. Voici l'essentiel du passage qui précède la retranscription d'un fragment du Journal :

Et quand, avant d'éteindre la bougie, je lus le passage que je transcris plus bas, mon absence de dispositions pour les lettres, pressentie jadis du côté de Guermantes, confirmée durant ce séjour dont c'était le dernier soir - ce soir des veilles de départ où l'engourdissement des habitudes qui vont finir cessant, on essaie de se juger - me parut quelque chose de moins regrettable, comme si la littérature ne révélait pas de vérité profonde; et en même temps il me semblait triste que la littérature ne fût pas ce que j'avais cru. D'autre part, moins regrettable me paraissait l'état maladif qui allait me confiner dans une maison de santé, si les belles choses dont parlent les livres n'étaient pas plus belles que ce que j'avais vu. Mais par une contradiction bizarre, maintenant que ce livre en parlait j'avais envie de les voir. (Proust, 1990, p. 15)

Le narrateur confirme sa déception concernant ses orientations littéraires, tout en soulignant une déception nouvelle : celle de l'impuissance de la littérature. Néanmoins, les lignes qui suivent le passage du Journal renversent quelque peu la situation, en nous la présentant sous un angle plus favorable, du moins, en nous laissant entrevoir quelques échappatoires. Au final, le narrateur aura tout de même un certain espoir en la littérature, car il arrivera à en tisser les lois et les analogies, ce qui lui permet de cerner la nature de ses futurs talents d'écrivain, même s'il ne croira en ses talents qu'à la fin du Temps retrouvé lors des passages de «L'adoration perpétuelle» et du «Bal de têtes ». Après la scène du Journal, le narrateur remarque qu'il ne sait ni écouter ni observer, contrairement aux auteurs de ce 
"prestige de la littérature» (p. 23). Les Goncourt et lui ont pourtant fréquenté les mêmes gens (les Verdurin, les Cottard, Brichot, etc.), mais, alors que les deux frères écrivains considèrent ces gens comme fins, intelligents, ingénieux, voire géniaux, le narrateur, lui, remarque «les vulgarités sans nombre dont chacun était composé » (p. 24). La vision avec laquelle les Goncourt perçoivent et représentent le monde n'est pas la même que celle du narrateur, leur façon de faire fonctionner la littérature ne peut également qu'en être différente. Cette conclusion déplaît d'emblée au narrateur (car les Goncourt sont de grands écrivains), et il souhaite revoir ses anciennes fréquentations mondaines afin de saisir leur richesse individuelle qu'avaient laissé bruire certaines lignes du Journal. Toutefois, ce désir est de courte durée : il arrivera à se rassurer. Moment capital de la Recherche (que nous nous permettons de citer longuement), sans encore arriver à la révélation finale, le narrateur laisse poindre l'idée d'une conception nouvelle de la littérature, et c'est de cette manière (une fois peaufinée) qu'il devra bâtir son œuvre :

Je résolus de laisser provisoirement de côté les objections qu'avaient pu faire naître en moi contre la littérature les pages de Goncourt [...]. Même en mettant de côté l'indice individuel de naïveté qui est frappant chez ce mémorialiste, je pouvais d'ailleurs me rassurer à divers points de vue. D'abord, en ce qui me concernait personnellement, mon incapacité de regarder et d'écouter, que le journal cité avait si péniblement illustrée pour moi, n'était pourtant pas totale. Il y avait en moi un personnage qui savait plus ou moins bien regarder, mais c'était un personnage intermittent, ne reprenant vie que quand se manifestait quelque essence générale, commune à plusieurs choses, qui faisait sa nourriture et sa joie. Alors le personnage regardait et écoutait, mais à une certaine profondeur seulement, de sorte que l'observation n'en profitait pas. Comme un géomètre qui dépouillant les choses de leurs qualités 
sensibles ne voit que leur substratum linéaire, ce que racontaient les gens m'échappait, car ce qui m'intéressait, c'était non ce qu'ils voulaient dire mais la manière dont ils le disaient, en tant qu'elle était révélatrice de leur caractère ou de leurs ridicules; ou plutôt c'était un objet qui avait toujours été plus particulièrement le but de ma recherche parce qu'il me donnait un plaisir spécifique, le point qui était commun à un être et à un autre. Ce n'était que quand je l'apercevais que mon esprit - jusque-là sommeillant, même derrière l'activité apparente de ma conversation dont l'animation masquait pour les autres un total engourdissement spirituel - se mettait tout à coup joyeusement en chasse, mais ce qu'il poursuivait alors - par exemple l'identité du salon Verdurin dans divers lieux et divers temps -était situé à mi-profondeur, au-delà de l'apparence elle-même, dans une zone un peu plus en retrait. Aussi le charme apparent, copiable, des êtres m'échappait parce que je n'avais pas la faculté de m'arrêter à lui, comme un chirurgien qui, sous le poli d'un ventre de femme, verrait le mal interne qui le ronge. J'avais beau dîner en ville, je ne voyais pas les convives, parce que, quand je croyais les regarder, je les radiographiais.

Il en résultait qu'en réunissant toutes les remarques que j'avais pu faire dans un dîner sur les convives, le dessin des lignes tracées par moi figurait un ensemble de lois psychologiques où l'intérêt propre qu'avait eu dans ses discours le convive ne tenait presque aucune place. Mais cela enlevait-il tout mérite à mes portraits puisque je ne les donnais pas pour tels? Si l'un, dans le domaine de la peinture, met en évidence certaines vérités relatives au volume, à la lumière, au mouvement, cela fait-il qu'il soit nécessairement inférieur à tel portrait ne lui ressemblant aucunement de la même personne, dans lequel mille détails qui sont omis dans le premier seront minutieusement relatés - deuxième portrait d'où l'on pourra conclure que le modèle était ravissant tandis qu'on l'eût cru laid dans le premier, ce qui peut avoir une importance documentaire et même historique, mais n'est pas nécessairement une vérité d'art. (p. 24-25) 
Ici, Proust commence à révéler le fonctionnement de la vocation littéraire de son narrateur, en mettant en avant l'éventualité d'une analogie tout à fait nouvelle entre l'art et la vie, un rapport original allant à l'encontre de l'«importance documentaire et même historique » qu'orchestre la littérature anecdotique ou réaliste, flambeau du XIXe siècle français. La déception première du narrateur devant le Journal se change ainsi en ce qui deviendra une théorie sur l'art et sur la vie, sur le style et sur le vécu. Ce passage nous laisse deviner que l'art ne crée pas ex nihilo, mais a bel et bien besoin de la vie pour arriver à tisser des lois générales, les « vérités d'art » dont parle Proust. La déception éprouvée à la suite de la lecture du Journal transformera le narrateur en voyant (d'où la « radiographie » qu'il évoque) : il sait maintenant que le monde possède quelque chose de trop grand et de caché, et que ce quelque chose ne peut se traduire et se transmettre que par l'art ou, plutôt, par une forme nouvelle de l'œuvre d'art, "rapport unique» qu'il devra décrypter. Proust critique l'observation des Goncourt par ce procédé qu'il nomme la traduction ou la transcription, engrenage essentiel de l'autofiction.

L'apprentissage du narrateur de la Recherche fonctionne d'une curieuse manière, les déceptions y jouant souvent un rôle plus important que les succès. Cependant, d'un autre point de vue, on pourrait affirmer que toutes ces épreuves ont bel et bien été couronnées de succès, parce que ratées ${ }^{2}$. Les viles

\footnotetext{
2 Imaginons un instant ce qu'aurait pu être la vie du narrateur: il serait devenu complaisant envers ses souvenirs plutôt que de les interroger et de les travailler; il aurait sans doute été un grand mondain, comme Swann ou Charlus, incapable de toute production littéraire, étant un collectionneur, préférant la conversation au réel travail de l'artiste (qui est un travail solitaire, on se souvient de la belle formule de Proust, dans le Contre SainteBeuve: "les livres sont les enfants du silence»); il se serait marié avec
} 
tentations ont été écartées, certes au prix de quelques déceptions, mais, somme toute, le narrateur a bien réussi le pari qu'il s'était donné sans le savoir vraiment: devenir écrivain, trouver son sujet, lequel, au final, sera sa propre vie.

\section{Vocation : autofiction}

L'autofiction, contrairement à l'autobiographie, aux Mémoires et aux Souvenirs, n'est pas l'affaire du détail, aussi scrupuleux soit-il. Avec l'autofiction, le critère de base n'est plus la ressemblance, l'observation ou la flatterie. Parlant de Proust et de l'entreprise de sa Recherche, Grimaldi, vers la fin de son ouvrage, nous dit à peu près la même chose :

Cette pathétique intensité et ce chatoiement du réel, tels que nos sensations nous les avaient fait vivre, comment les restituer? Tel qu'en use la "connaissance conventionnelle» que nous prenons des choses, il va de soi que le commun langage en est incapable. Répudiant les lieux communs, les stéréotypes, les expressions toutes faites, il faut donc inventer une nouvelle qualité du langage qui puisse faire imaginer ce que chaque moment avait eu de plus pathétique et chaque sensation d'essentiel. Il faut pour cela traduire la singularité de ce que nous avons vécu, autrement dit procéder à une véritable transmutation qui fasse reconnaître dans l'enchaînement et l'association des mots la qualité de nos souvenirs. Rien qu'une opération alchimique pourra donc faire exprimer par une

\footnotetext{
Albertine, ce qui lui aurait donné une vie de soupçons, il serait demeuré captif de sa jalousie et de sa relation, au mieux il s'y serait habitué, comme Swann avec Odette; il aurait accepté la Venise réelle, avec tous ses mensonges et toutes ses fables, traquant à l'infini des jeunes filles lui faisant vaguement penser à Albertine, une Albertine encore joyeuse, que le mystère n'aurait pas rendue inaccessible; il aurait peut-être écrit ses Mémoires, voire son autobiographie, à la manière des Goncourt, devenant un fin observateur de la surface des choses, étant incapable de cerner toute loi générale qui lui aurait permis de constater qu'une autre littérature est possible.
} 
langue que tous puissent entendre des sensations que nul ne peut partager. (2008, p. 230-231)

Proust est peut-être le premier à affirmer aussi fortement que la littérature dite personnelle doit rejeter le réalisme d'observation, le réalisme d'anecdote, la perfection d'un beau style comme celle d'une vie modèle. On peut noter les commentaires de Walter Benjamin qui, dès 1929, arrive à cerner les principaux enjeux de la poétique proustienne, nous informant ainsi sur la place qu'occupe dans la Recherche ce que l'on nommera plus tard autofiction :

Les treize volumes de la Recherche sont le produit d'une inconstructible synthèse où se rencontrent, pour former un ouvrage autobiographique, l'oubli de soi du mystique, l'art du prosateur, la verve du satiriste, le savoir de l'érudit et le parti pris du monomane. On a dit avec raison que toute grande œuvre littéraire inaugure un genre, ou le dissout, en un mot est un cas spécial. Mais parmi ces cas spéciaux, celui-ci est l'un des plus insaisissables. À commencer par la structure, qui unit la fiction, les mémoires et le commentaire, jusqu'à la syntaxe avec ses phrases sans rivages (ce Nil du langage qui déborde ici, pour les fertiliser, sur les plaines de la vérité), tout ici échappe à la norme. Que ce grand cas singulier de la littérature en constitue en même temps la plus importante réussite des dernières décennies, c'est la première constatation, fort instructive, qui s'impose à l'observateur. Et malsaines au plus haut point sont les conditions qui lui ont servi de base. Une maladie rare, une richesse peu commune, des penchants anormaux. Tout dans cette vie n'est pas parfait, mais tout y est exemplaire. À cette réussite littéraire de premier ordre, tout cela assigne son lieu au cœur de l'impossible, au centre, et certes en même temps au point d'indifférence, de tous les dangers, et caractérise la grande réalisation de cette " œuvre d'une vie " comme la dernière avant longtemps. L'image de Proust est la plus haute expression physiognomonique que pouvait atteindre l'écart croissant entre la littérature et la vie. (2000, p. 135-136) 
Une vie, même "malsaine », peut devenir une œuvre d'art, car l'autofiction ne cherche pas la même chose que l'autobiographie : non plus le sens d'une vie, mais son usage.

\section{Proust et l'autofiction}

La Recherche de Proust met en scène un personnage qui, sans le savoir d'emblée, entreprend un apprentissage qui s'échelonnera sur toute sa vie. Ses déceptions l'éloigneront un temps de la vie en plus de lui donner la vision d'une forme neuve de l'œuvre d'art, c'est-à-dire que la vérité ne doit plus être racontée, observée ou annotée, mais bien créée, produite ou traduite. Cette nouvelle conception de l'œuvre d'art octroie au personnage proustien un nouveau regard sur sa propre vie qui, du coup, n'est plus décevante. On ne peut pas le dire mieux que Proust lorsqu'il insiste sur l'analogie entre la Recherche et un instrument: l'ouvrage de l'écrivain n'est qu'une espèce d'instrument d'optique qu'il offre au lecteur afin de permettre de discerner ce que, sans ce livre, il n'eût peut-être pas vu en lui$m e ̂ m e^{3}$. Et qu'est-ce donc que l'autofiction, telle que l'avait prévue Proust, sinon l'apprentissage d'un instrument permettant une nouvelle lecture de soi? Mais cet apprentissage du narrateur proustien n'est pas gratuit, c'est-à-dire qu'il ne se fait pas dans le vide : pour avoir une révélation sur la vie, à la fin du parcours, il faut bien sûr avoir vécu, avoir vécu quelque chose de particulier. Cette chose particulière, Anne Henry la

\footnotetext{
3 Nous paraphrasons ce passage du Temps retrouvé: "Ils ne seraient pas, selon moi, mes lecteurs, mais les propres lecteurs d'eux-mêmes, mon livre n'étant qu'une sorte de ces verres grossissants comme ceux que tendait à un acheteur l'opticien de Combray; mon livre, grâce auquel je leur fournirais le moyen de lire en eux-mêmes » (p. 338).
} 
nomme crise du sujet. C'est sur ce concept qu'il faut faire la lumière.

\section{La crise proustienne du sujet : entre banalité et agencement}

Avec La tentation de Marcel Proust, Henry illumine ce qui est, selon nous, l'enjeu primordial de la Recherche :

La tentation de Marcel Proust n'est autre que celle d'un acquiescement à la crise du sujet, ce fleuron du nihilisme moderne. Sous le velours de ses phrases et l'amabilité de ses anecdotes, ̀̀ la recherche du temps perdu n'est pas un livre paisible. Un drame majeur s'y déroule, qu'aucun romancier n'avait imaginé jusque-là et pas davantage n'aurait su conter, en lui assurant, sans le nommer, une telle crédibilité, en l'inscrivant, pour plus de pureté, dans une existence quotidienne si peu menacée au-dehors [...].

Dès la première page [...] une défiance se dessine, gâte la spontanéité des gestes les plus simples. Elle cristallise autour d'un point précis : bien que le protagoniste soit cloué au centre de sa propre histoire, il ne peut la gouverner à son gré [...]. Cette opacité décourageante provoque en lui la tentation par excellence, le désaveu de la vie - une vie qui ne demandait qu'à aimer. (2000, p. 1-2)

Cette notion de crise du sujet ne peut qu'être en profonde correspondance avec l'esthétique et l'essence de l'autofiction. En fait, à relire l'œuvre proustienne, on remarque dès les premières pages un sujet littéralement en pleine crise, tourmenté par un curieux malaise, celui de la fragmentation de son être dans le «kaléidoscope de l'obscurité » (Proust, 1988, p. 4). Fragmentation, mais aussi multiplication de lui-même: "Quelquefois, comme Ève naquit d'une côte d'Adam, une femme naissait pendant mon sommeil d'une fausse position de ma cuisse» (p.4). En somme, morcellement identitaire et 
corporel, mêlé d'une incompréhension du monde extérieur. Dès lors, voici un autre passage célèbre du début de la Recherche, qui gagne à être relu avec un point de vue différent, celui de la crise du sujet propre à la modernité et qui deviendra une des raisons d'être de l'existence de l'autofiction :

Quand je me réveillais ainsi, mon esprit s'agitant pour chercher, sans y réussir, à savoir où j'étais, tout tournait autour de moi dans l'obscurité, les choses, les pays, les années. Mon corps, trop engourdi pour remuer, cherchait, d'après la forme de sa fatigue, à repérer la position de ses membres pour en induire la direction du mur, la place des meubles, pour reconstruire et pour nommer la demeure où il se trouvait. Sa mémoire, la mémoire de ses côtes, de ses genoux, de ses épaules, lui présentait successivement plusieurs des chambres où il avait dormi, tandis qu'autour de lui les murs invisibles, changeant de place selon la forme de la pièce imaginée, tourbillonnaient dans les ténèbres. (p. 6)

On pourrait être tenté d'expliquer cette petite crise du sujet de façon narrative, en évoquant l'insomnie ou les troubles qui habitent le sommeil du protagoniste. Néanmoins, nous jugeons cette explication un peu simple; il faut investiguer pour arriver à faire ressortir l'inquiétante étrangeté qui traverse cette scène. Dans ce qui est le véritable drame du coucher de la Recherche, le personnage proustien se remémore, divague sur son être, sur le monde et sur le temps. Voilà une première manifestation de sa folie ou de sa crise, les premiers signes de son malaise et le premier accroc dans le fonctionnement de son être-au-monde. C'est pourquoi la symbolique qui entoure cette scène, comprise dans toute la modernité qu'elle revendique, est significative pour l'esthétique globale de la Recherche. Elle souligne le problème proustien par excellence. 
L'apprentissage du narrateur répond à un problème aussi grave que moderne, celui de la crise du sujet: l'identité n'est plus fixe, elle est maintenant en mouvance, en constant devenirautre. Proust, par sa nouvelle conception du sujet (et des sujets, comme on dit des histoires), ferme la porte des grandes fresques historiques et romanesques du XIX ${ }^{\mathrm{e}}$ siècle (Balzac, Zola, pour ne nommer que les plus connus), et fait entrer la littérature française dans sa modernité. Avec l'autofiction, plus besoin d'avoir un bon sujet ou même d'être un bon sujet, car la vérité est ailleurs.

Comme l'écrit Henry, "[i]l est impossible d'évoquer le moindre aspect du récit proustien si on le sépare du perfectionnement d'une impasse existentielle» (2000, p. 48). Mais quel est le résultat de cette «impasse existentielle », sens unique que semble avoir pris le narrateur dans son long trajet rythmé par une pléiade quasi innombrable de déceptions? Notre réponse, comme celle de Henry: le nihilisme proustien. Nihilisme dans un monde plat auquel le narrateur ne peut pas s'agripper, un monde asignifiant. Mais attention, Proust a également trouvé un remède original au mal-être qui ronge son narrateur. La découverte de ce remède incarne le début de la vita nova du héros proustien comme de celle de son créateur. C'est la grande extase du Temps retrouvé, début de la fin de cette étrange histoire d'une vocation qu'est la Recherche :

Le Temps retrouvé est le théâtre d'une révélation extraordinaire : au terme d'un récit qui détaille avec minutie les étranglements auxquels l'existence a soumis un être qu'appesantit de surcroît une indifférence lasse due à la décrue de sa force, c'est la fulguration, le grand retournement qui efface doutes et "calme plat du désespoir», comme disait Baudelaire. Après avoir trébuché sur le pavé de la cour des Guermantes, le protagoniste accède à un univers nouveau où 
rien n'est pourtant changé si ce n'est le regard qu'il porte sur ce qu'il condamnait comme vie mauvaise. Il n'est pas transporté au septième ciel, il n'a pas été visité par un ange, il demeure sur le sol phénoménal et c'est sans ébriété qu'une fois remis de son étonnement il annonce seulement quelle activité sera la sienne désormais : fixer son acceptation de la vie dans les conditions où celle-ci a été vécue. Ainsi prend fin la tentation nihiliste. (Henry, 2000, p. 147)

La crise du sujet et le nihilisme qui sévissent dans la Recherche donnent ainsi à l'art un mandat nouveau : l'art doit maintenant être producteur de vie et créateur de vérité. Et les feux de la rampe brillent sur l'autofiction. En effet, celle-ci est cette forme originale de l'œuvre d'art qui opère et permet une nouvelle conception de l'identité: une identité qui ne peut être pleinement rendue (comme on dit en cinéma) que par l'œuvre d'art elle-même. Boucle vertigineuse du néologisme doubrovskien : pour un mal moderne (crise du sujet, impasse identitaire), il faut un remède nouveau (autofiction). La Recherche orchestre la création d'une nouvelle ipséité pour combattre le nihilisme des Temps modernes. L'identité, avec Proust, quitte peu à peu le social et devient finalement redevable à l'art. C'est pourquoi l'autofiction est l'orchestration de notre moi profond, pas de notre moi social. L'identité sociale prend racine dans un espace quadrillé (mœurs, us et coutumes, lois, morale, bien et mal, codes, etc.), alors que l'identité narrative (fictionnelle ou artistique) se développe sur un espace lisse (que l'Habitude n'a pas encore quadrillé). Espace quadrillé de l'autobiographie, espace lisse de l'autofiction, pourrait-on dire.

Nous voudrions maintenant attirer l'attention sur un aspect plutôt curieux de l'entreprise proustienne, son rapport avec la banalité. Somme toute, l'expérience du narrateur peut, 
dans ses grandes lignes, être qualifiée de quelconque, n'ayant vraiment rien d'héroïque ou de particulièrement romanesque, comme nous l'avons déjà souligné. Le constat de Henry alimente cette hypothèse : "C'est à partir [des] petites choses que tout se joue. Dans ce reclassement hiérarchique, n'est-il pas aussi important de goûter au sommeil en wagon-lit que d'être présenté à une altesse?» (2000, p. 107. Nous soulignons). Le verdict de Proust est sans équivoque: les événements euxmêmes ne sont rien. La trame de la Recherche, à savoir l'apprentissage de son narrateur, est construite sur des riens, même si on peut y déceler une certaine forme de romanesque. Que fait le narrateur? Quels sont les événements marquants de sa vie? Quels sont les préludes à la découverte de sa vocation? Pas grand-chose, certes, mais regardons tout de même: il n'arrive pas à s'endormir; il trempe un gâteau dans une tasse de thé; il veut voir sa mère; il fixe trois arbres particuliers ou encore des aubépines; il dort dans une nouvelle chambre; il va pour la première fois au théâtre; il va à un dîner; il attache sa bottine; il voit deux jeunes filles danser; il écoute une sonate ou un septuor; il fait erreur sur la personne; il n'arrive pas à déchiffrer une signature; il lit un livre; il trébuche sur un pavé; il écoute une cuiller se cogner contre une tasse; il remarque la rigidité d'une serviette, etc. Pourtant, tous ces moments sont des moments clés de la Recherche et dans la vie de son protagoniste principal. Comment tout cela peut-il bien faire une œuvre? Aurait-on idée d'écrire son autobiographie si on avait vécu une vie pareille, constituée de banalités semblables? Proust nous prouve que la réponse peut être oui, mais, pour y arriver, il faut instaurer une analogie nouvelle entre la vie et l'œuvre d'art. 
Par ce « reclassement hiérarchique » évoqué par Henry, Proust devient probablement le premier auteur à mettre autant en avant l'idée des moments ou instants quelconques. Reclassement qui bouleverse les rapports préexistants entre l'art (surtout la littérature) et la vie. Ainsi, la banalité proustienne devient ni plus ni moins un nouveau rapport au réel, et l'instant quelconque est alors un point de vue privilégié. Tout le mérite de Proust est là : avoir su construire une fresque romanesque - cathédrale, robe ou toile - en rejetant ce que l'on considère généralement comme des moments privilégiés, mais, en revanche, à partir de moments quelconques. Et cette différence de point de vue est également celle qui oppose l'autobiographie à l'autofiction : là où la première ne repose que sur des instants privilégiés (du moins pour les autobiographies classiques), la seconde accepte et travaille l'instant quelconque, la contingente banalité de notre vie.

En outre, cette théorie des instants quelconques rapproche la Recherche de l'esthétique propre à l'art du cinématographe, quoi qu'en dise Proust qui (particulièrement dans le Temps retrouvé) n'était pas tendre à l'égard du septième art. Deleuze (auteur de Proust et les signes), au début de l'Image-mouvement, est très clair là-dessus. Le philosophe $\mathrm{y}$ reprend les idées d'un autre penseur, soit les thèses d'Henri Bergson sur le mouvement, thèses qui en viennent à opposer la science antique à la science moderne. Le septième art appartient à juste titre à cette révolution dans le mouvement qui est la découverte du moment quelconque (déclassant ainsi la supériorité du moment privilégié) :

La révolution scientifique moderne a consisté à rapporter le mouvement, non plus à des instants privilégiés, mais à l'instant quelconque. Quitte à recomposer le mouvement, on ne le 
recomposait plus, à partir d'éléments formels transcendants (poses), mais à partir d'éléments matériels immanents (coupes). (Deleuze, 1983, p. 13)

Pour les deux philosophes, cette révolution qui amena le mouvement dans sa modernité a enclenché une évolution créatrice qui englobe aussi bien l'astronomie moderne (Kepler), la physique moderne (Galilée), la géométrie moderne (Descartes) que le calcul infinitésimal (Newton et Leibniz). C'est maintenant la succession mécanique d'instants quelconques qui remplace l'ordre dialectique des poses. Ici, le cinéma, « dernierné de cette lignée dégagée par Bergson », entre en jeu :

On pourrait concevoir une série de moyens de translation (train, auto, avion...), et parallèlement une série de moyens d'expression (graphique, photo, cinéma): la caméra apparaîtrait alors comme un échangeur, ou plutôt un équivalent généralisé des mouvements de translation [...]. Quand on s'interroge sur la préhistoire du cinéma, il arrive qu'on tombe dans des considérations confuses, parce qu'on ne sait pas où faire remonter ni comment définir la lignée technologique qui le caractérise. Alors on peut toujours invoquer les ombres chinoises ou les systèmes de projection les plus archaïques. Mais, en fait, les conditions déterminantes du cinéma sont les suivantes : non pas seulement la photo, mais la photo instantanée (la photo de pose appartient à l'autre lignée); l'équidistance des instantanés; le report de cette équidistance sur un support qui constitue le « film » (c'est Edison et Dickson qui perforent la pellicule); un mécanisme d'entraînement des images (les griffes de Lumière). C'est en ce sens que le cinéma est le système qui reproduit le mouvement en fonction $d u$ moment quelconque, c'est-à-dire en fonction d'instants équidistants choisis de façon à donner l'impression de continuité. (Deleuze, 1983, p. 13-14)

Même un cinéaste comme Sergei Eisenstein, dans ses films historiques à dimension épique (Le cuirassé Potemkine, Alexandre Nevsky, Ivan le terrible), se doit de travailler avec des 
instants quelconques afin de reconstituer le moment privilégié du drame ou de l'épopée, et ce n'est absolument pas un paradoxe :

Les instants privilégiés d'Eisenstein, ou de tout autre auteur, sont encore des instants quelconques; simplement, l'instant quelconque peut être régulier ou singulier, ordinaire ou remarquable. Qu'Eisenstein sélectionne des instants remarquables n'empêche pas qu'il les tire d'une analyse immanente du mouvement, pas du tout d'une synthèse transcendante. (p. 15)

En bref, le moment privilégié ou singulier n'est qu'une des possibilités de l'instant quelconque. Et c'est ainsi que Deleuze définit le cinéma comme le système qui reproduit le mouvement en le rapportant à l'instant quelconque. Fascination de Louis Lumière (et de ses opérateurs) pour les foules, les passants, les moyens de transport, une rue, un cheval, un déjeuner de bébé, un couple, un marché, un vêtement... Catalyser toute la force de l'impressionnisme par la vigueur de l'instant quelconque. Il s'agit alors, comme le dit Henry à propos de la Recherche, d'un «fief anecdotique nouveau ». Chez Proust la vie est là : petits instants, moments anodins, possible banalité de notre vie. L'œuvre d'art telle que Proust la conçoit et la défend nous permet «d'assigner une place au moindre petit fait » (Henry, 2000, p. 204) et d'opérer une « reconstruction de la vie sur le plan même de la vie » (p. 208).

Proust ne défend pas la banalité pour la banalité. Il doit y avoir une façon d'éviter l'impasse de la crise du sujet baignant dans les instants quelconques, en plus de justifier la nouvelle forme de romanesque que nous venons d'évoquer. La réponse se trouve (quoique sommairement) dans l'ouvrage de Henry, d'abord par le constat suivant : 
[Proust] ne peut raconter tout bonnement, comme Balzac ou Zola, une histoire où la succession événementielle suffit à tout. Si l'acte si simple, embrasser une jolie fille, doit faire avancer la réflexion sur des plans aussi différents que la femme et le lieu, l'évolution du langage d'une jeune bourgeoise, la traîtrise des domestiques, la bonté sous-jacente à l'érotisme pur, l'inadaptation de l'homme au baiser et le caractère impressionnant des modifications de perspective, l'écrivain est bien obligé de recourir à une ruse littéraire pour faire admettre un ensemble hétéroclite, si l'on s'en tient aux critères traditionnels. (p. 199-200)

Cette «ruse littéraire», Henry la nomme en reprenant un concept proprement cinématographique, à savoir le montage :

Proust est ainsi conduit à inaugurer cette technique moderne : le montage, c'est-à-dire une continuité artificielle obtenue par ajustage de séquences fabriquées indépendamment les unes des autres, relevant de domaines différents mais recevant une unité supérieure du projet général [...]. Son organisation assure un nouveau style de narration infiniment plus complexe que la structure, si parfaite soit-elle, de L'Éducation sentimentale fondée sur un ressassement rythmique qui respecte la tradition. La force de ce montage vient de ce qu'il ne vise pas à des effets esthétiques, alternance, symétrie, etc., mais qu'il est mis au service d'une démonstration. Comme on dit en cinéma, c'est un montage idéologique. (p. 200)

C'est de cette manière que la comparaison de la Recherche avec une robe (comme le fait le narrateur vers la fin du Temps retrouvé) peut prendre toute sa signification: l'œuvre proustienne est littéralement collée, morceau par morceau, morceau quelconque par morceau quelconque, car chacune des pièces fait partie d'une idée-non pas narrative mais esthétique - qui l'englobe et lui donne un sens nouveau. Cette idée de montage ou d'agencement illumine aussi plusieurs enjeux primordiaux de l'œuvre proustienne: la rencontre des deux côtés (côté de chez Swann, côté de Guermantes), les 
surprenantes métaphores proustiennes ou encore la mémoire involontaire établissant un lien et donc un agencement entre deux moi bien distincts dans le temps et dans l'espace. Avec Proust, c'est toujours la vie qui écrit le livre, chacun porte en soi son livre, il suffit d'en trouver la clé, l'idée suprême qui en dicte les lois et l'idéologie derrière le montage.

\section{Montage des identités}

La conception de l'identité proustienne encourage la transcription, la traduction et l'agencement de toute une vie aussi fragmentée soit-elle-dans une œuvre d'art. L'agencement donne un sens au rhizome de notre vie et octroie à l'écrivain une nouvelle façon de dire «je » qui n'exclut pas la multiplicité (notre multiplicité) : montage des identités, montage qui permet de combattre le trop grand de la vie moderne, car l'artiste y opère une fragmentation idéologique. Le moment (quelconque) ne se vaut plus pour lui-même, mais fait maintenant partie intégrante de la machine littéraire proustienne : il participe à son agencement, est directement lié aux lois générales que l'œuvre tisse et monte sous nos yeux. C'est justement cela, la découverte du Temps retrouvé : après la révélation finale, le vécu du narrateur repasse dans une sorte de zone, et tout devient (ou plutôt deviendra) solennel et, surtout, significatif, car les lois générales ont été tissées et la vérité a été produite et découpée. C'est la grande joie du perspectivisme et de l'agencement proustiens, en réponse (mais en même temps redevable) à la curieuse crise du sujet que vit le narrateur. La vie est alors un rhizome de possibilités qui se valent toutes, car leur salut dépend de la construction d'un agencement à venir. L'autofiction proustienne rend 
hommage à la «toute-puissance de l'existence » (Henry, 2000, p. 29), puissance découverte par le montage idéologique mis en marche par cette nouvelle conception de l'œuvre d'art.

L'autofiction travaille notre identité par montage, alors que l'autobiographie se contente d'une simple image, d'un cliché, d'un ça-a-été (pour reprendre le mot de Roland Barthes dans La Chambre claire) plutôt que d'un ça-devient mouvement perpétuel de l'autofiction et de l'écriture proustienne.

\section{Bibliographie}

BARTHES, Roland. (1980), La Chambre claire : note sur la photographie, Paris, Cahiers du cinéma.

BENJAMIN, Walter. (2000 [1929]), «L'image proustienne », dans CEuvres II, Paris, Gallimard, coll. « Folio Essais », p. 135-155.

Colonna, Vincent. (2004), Autofiction \& autres mythomanies littéraires, Auch, Tristram;

—. (1989), «L'autofiction : essai sur la fictionnalisation de soi en littérature ", thèse de doctorat de l'École des hautes études en sciences sociales, sous la direction de Gérard Genette, $<$ http://tel.archives-ouvertes.fr/docs/00/04/70/04/PDF/tel00006609.pdf> 
DeleuZE, Gilles. (1983), Cinéma 1, L'Image-mouvement, Paris, Minuit, coll. « Critique »;

—. (1985), Cinéma 2, L’Image-temps, Paris, Minuit, coll. « Critique ».

DouBRovsKY, Serge (2001 [1977]), Fils, Paris, Gallimard, coll. « Folio »;

-. (1988), "Corps du texte/texte du corps», dans Autobiographiques: de Corneille à Sartre, Paris, PUF, coll. «Perspectives critiques », p. 43-60.

GASPARINI, Philippe. (2008), Autofiction : une aventure du langage, Paris, Seuil, coll. «Poétique ».

GenetTe, Gérard. (1992 [1982]), Palimpsestes : la littérature au second degré, Paris, Seuil, coll. « Points Essais ».

GRIMALDI, Nicolas. (2008), Proust, les horreurs de l'amour, Paris, PUF, coll. « Perspectives critiques ».

Henry, Anne (2000), La tentation de Marcel Proust, Paris, PUF, coll. « Perspectives critiques».

Proust, Marcel. (1988 [1913]), Du côté de chez Swann, Paris, Gallimard, coll. « Folio Classique »;

—. (1990 [1927]), Le Temps retrouvé, Paris, Gallimard, coll. « Folio Classique ». 


\title{
Résumé
}

À la recherche du temps perdu est l'histoire d'une crise identitaire, celle d'un sujet qui souhaite écrire, mais n'y arrive pas. Au Temps retrouvé, c'est la révélation finale : le narrateur a enfin compris certaines lois, qu'il devra observer et traduire avec son "télescope », c'est-à-dire avec son œuvre d'art entendue comme instrument ou comme machine. Ainsi, le personnage proustien est contraint à créer un dispositif original pour parler de soi, une nouvelle herméneutique du sujet, ce qui fait de la Recherche la première vraie autofiction avant la lettre. L'autofiction proustienne, par son travail sur notre «moi profond", combat la crise identitaire et le nihilisme pour proposer un nouveau montage des identités.

\begin{abstract}
Remembrance of things past is the story of an identity crisis, that of a subject who wishes to write, but does not succeed. With Time regained, it is the final revelation: the narrator finally understood certain laws, that he will have to observe and translate with his "telescope", that is with his work of art, taken as an instrument or a machine. Thus, the proustian character is forced to create an original device to tell about oneself, a new hermeneutics of the subject, which makes Remembrance of things past the first true autofiction, before its time. The proustian autofiction, by its work on our "inner self", fights identity crisis and nihilism to propose a new editing of the identities.
\end{abstract}

